\title{
Molecular pharmacology on insect biogenic amines*
}

\author{
Hiroto OHTA** \\ Graduate School of Science and Technology, Kumamoto University, \\ 2-39-1 Kurokami, Kumamoto 860-8555, Japan
}

(Accepted May 20, 2009)

\begin{abstract}
Octopamine, tyramine and dopamine are physiologically important biogenic amines in insect peripheral and nervous tissues. The interactions of these amines with their specific receptors primarily up- or downregulate adenylate cyclase via the $\mathrm{G}_{\mathrm{s}}$ or $\mathrm{G}_{\mathrm{i}}$ subunit, respectively, leading to an increase or decrease in the levels of the second messenger cAMP within cells. These G-protein-coupled receptors are attractive targets for insecticides. This study performed functional and pharmacological characterizations of each biogenic amine receptor using heterologous expression systems for the cloned receptors of the silkworm, Bombyx mori. Such study may aid in the development of specific insecticides or insectistatics that target biogenic amine receptors. (C) Pesticide Science Society of Japan
\end{abstract}

Keywords: biogenic amines, receptor, octopamine, tyramine, dopamine, Bombyx mori.

\section{Introduction}

Biogenic amines found in the peripheral and nervous tissues of invertebrates such as insects function as neurotransmitters, neuromodulators and neurohormones, and regulate diverse physiological events. Octopamine (OA) and tyramine (TA) are typical phenolic biogenic amines in insects. Dopamine (DA) is also a typical biogenic amine, but it is thought to be the only neuroactive catecholamine in insects, whereas vertebrates also have adrenaline and noradrenaline.

The interactions of OA, TA, and DA with their specific receptors primarily up- or downregulate adenylate cyclase via the $\mathrm{G}_{\mathrm{s}}$ or $\mathrm{G}_{\mathrm{i}}$ subunit, respectively, leading to an increase or decrease in the levels of the second messenger cAMP within cells. These G-protein-coupled receptors are attractive targets for insecticides. For example, demethylchlordimeform (the in vivo metabolite of the formamidine insecticide chlordimeform) acts as an OA receptor agonist, exerting pesticidal and pestistatic effects.

To analyze the structure-activity relationships of 2phenylethylamines and 5-phenyloxazoles in a quantitative manner, we performed cAMP assays using head homogenates from fifth instar larvae of the silkworm, Bombyx mori. This

* See Part II for the full Japanese article.

** To whom correspondence should be addressed.

E-mail: hiohta@gpo.kumamoto-u.ac.jp

(C) Pesticide Science Society of Japan should in turn lead to the identification of physiological and biochemical tools, facilitate the development of agrochemicals, and provide structural information regarding the ligandbinding sites of biogenic amine receptors. However, there is a large problem with this assay system; the presence of multiple receptor types (i.e., OA, TA, and DA receptors) in the homogenates means that the positive or negative modulation of adenylate cyclase in a substituent-dependent fashion is liable to be estimated as an integrated result. Therefore, to study the specific mechanisms underlying the positive versus negative modulation of adenylate cyclase by these ligands, thus providing more accurate data on structure-activity relationships, we need to examine the response to each ligand using expression systems for cloned biogenic amine receptors from B. mori.

In this study, to develop a novel system to evaluate compounds that target biogenic amine receptors, we cloned OA, TA, and DA receptor genes from B. mori, and expressed each receptor functionally in a cell line. The pharmacological analysis using the single-receptor expressing systems revealed functions of each cloned receptor, as well as structures and pharmacological properties of the ligand-binding sites. The results are summarized here.

\section{Molecular cloning and characterization of the Bombyx mori tyramine receptor}

Cloning of the cDNAs for the OA, TA, and DA receptors of B. mori has not been reported, except for B96Bom, which was reported to encode a putative $B$. mori OA receptor with nega- 
tively modulating function toward adenylate cyclase; however, a functional characterization of the receptor was not attempted. Therefore, we cloned the cDNA, expressed it in a cell line, and examined receptor function.

A cDNA encoding a putative OA receptor cDNA, B96Bom, was isolated from Bombyx larvae, and the ligand response of the receptor stably expressed in human embryonic kidney (HEK)-293 cells was examined. At $0.1-100 \mu \mathrm{M}$, TA reduced forskolin-stimulated $(10 \mu \mathrm{M})$ intracellular cAMP levels by approximately $30-40 \%$. The antagonists yohimbine and chlorpromazine (each $10 \mu \mathrm{M}$ ) abolished the inhibitory effect of $1 \mu \mathrm{M}$ TA. Although OA also reduced cAMP levels, its potency was at least two orders of magnitude lower than that of TA. Furthermore, unlabeled TA $\left(\mathrm{IC}_{50}=5.2 \mathrm{nM}\right)$ inhibited specific $\left[{ }^{3} \mathrm{H}\right] \mathrm{TA}$ binding to the membranes of HEK-293 cells stably expressing the B96Bom receptor more potently than did OA $\left(\mathrm{IC}_{50}=1.4 \mu \mathrm{M}\right)$ or DA $\left(\mathrm{IC}_{50}=1.7 \mu \mathrm{M}\right)$. Combined with the results of a phylogenetic analysis, these findings indicate that the $\mathrm{B} 96 \mathrm{Bom}$ receptor is a B. mori TA receptor that is negatively coupled to adenylate cyclase.

\section{Responses of the cloned Bombyx tyramine receptor to 2-phenylethylamines and 5-phenyloxazoles}

The actions of several 2-phenylethylamines and 5-phenyloxazoles on the heterologously expressed Bombyx TA receptor were examined. 5-(4-Hydroxyphenyl)oxazole did not attenuate forskolin-stimulated cAMP production in HEK-293 cells expressing the TA receptor, although the compound bound to the receptor. 2-Phenylethylamines also inhibited $\left[{ }^{3} \mathrm{H}\right] \mathrm{TA}$ binding to the cloned TA receptor. Among the 2-phenylethylamines tested, 2-chloro-2-(4-chlorophenyl)ethylamine was the most potent inhibitor of $\left[{ }^{3} \mathrm{H}\right] \mathrm{TA}$ binding, with an $\mathrm{IC}_{50}$ of $30.4 \mathrm{nM}$. This compound acted as an antagonist and abolished the TA-induced attenuation of forskolin-stimulated cAMP production in the cloned TA receptor. The accumulation of additional information from similar studies would be useful for developing specific probes for TA receptors.

\section{Molecular cloning and characterization of a Bombyx mori octopamine receptor}

Because our results indicated that the B96Bom receptor was a TA receptor rather than an OA receptor, we searched for cDNA encoding a genuine OA receptor and isolated it from the nerve tissue of $B$. mori larvae. An amino acid sequence comparison showed that the Bombyx OA receptor is nearly identical to OA receptors isolated from Periplaneta americana (Pa oa1), Apis mellifera (AmOA1), and Drosophila melanogaster (OAMB or DmOA1A). The Bombyx OA receptor was stably expressed in HEK-293 cells. OA treatment at concentrations $>1 \mu \mathrm{M}$ led to an increase in intracellular cAMP concentrations. The OA receptor agonist demethylchlordimeform also elevated cAMP levels to the same maximal level (approximately 5-fold greater than basal levels) as that induced by OA. By contrast, the biogenic amines TA and DA and chlordimeform lacked effect. The OA-stimulated increase in cAMP levels was suppressed by antagonists; the rank order of antagonist activity was chlorpromazine $>$ mianserin $=$ yohimbine.

\section{Identification of the agonist-binding site of the tyramine receptor}

Information regarding the TA-binding site of TA receptors is helpful not only for performing structure-activity studies of TA receptor ligands, but also for identifying agonists that bind to TA receptors specifically. Therefore, we performed a series of site-directed mutagenesis experiments to identify amino acid residues in the $B$. mori TA receptor involved in the interaction with the amino and $p$-hydroxyl groups of TA.

To identify the amino acid residues interacting with the amino and $p$-hydroxyl groups of TA in the Bombyx TA receptor, several amino acid residues (Asp134, Ser218, Ser219, Ser220, and Ser222), which were predicted to be putative TAbinding sites from analogy with the agonist-binding site of the $\beta_{2}$-adrenergic receptor, were replaced with Ala. The ability of these mutant receptors, each of which was stably expressed in HEK-293 cells, to bind TA and attenuate forskolin-stimulated cAMP production in response to TA was examined experimentally.

The D134A TA receptor showed no specific $\left[{ }^{3} \mathrm{H}\right] \mathrm{TA}$ binding and no attenuation of the intracellular cAMP levels after treatment with TA, indicating that Asp134 in transmembrane domain III is an important residue for both interaction with TA and receptor activation. The carboxyl group of Asp134 probably forms an ion pair with the protonated amino group of TA.

The ability of TA to inhibit specific $\left[{ }^{3} \mathrm{H}\right] \mathrm{TA}$ binding to the S219A and S220A TA receptors was similar to that observed in the wild-type TA receptor. Although the S218A and S222A TA receptors showed no specific $\left[{ }^{3} \mathrm{H}\right] \mathrm{TA}$ binding, they were still able to mediate the attenuation of cAMP levels in response to a high TA concentration $(100 \mu \mathrm{M})$. By contrast, the double mutation of Ser218 and Ser222 to Ala led to the loss of the TA-induced attenuation of cAMP levels. These findings suggest that both Ser218 and Ser222 in transmembrane domain $\mathrm{V}$ contribute to the interaction with TA, as well as to receptor activation. Hydrogen bonds are probably formed between the hydroxyl groups of both Ser residues and the $p$-hydroxyl group of TA.

\section{Comparative analysis of the function and pharmacology of two D1-like dopamine receptors}

We cloned three DA receptors (BmDopR1, BmDopR2, and unpublished BmDopR3) from B. mori and identified BmDopR1 and BmDopR2 as D1-like receptors that activate adenylate cyclase to increase intracellular cAMP levels, using HEK-293 cells stably expressing BmDopR1 or BmDopR2. In further studies of the respective receptor-expressing cells, the dose response to DA and pharmacological properties were ex- 
amined using cAMP assays.

BmDopR1 showed a dose-dependent increase in cAMP levels at DA concentrations up to $10^{-7} \mathrm{M}$ with an $\mathrm{EC}_{50}$ of $3.30 \mathrm{nM}$, whereas BmDopR2 required $10^{-6} \mathrm{M}$ DA for activation. In BmDopR1-expressing cells, DA at $10^{-6}$ to $10^{-4} \mathrm{M}$ induced 30-50\% lower cAMP production than $10^{-7} \mathrm{M}$ DA. BmDopR2-expressing cells showed a standard sigmoidal dose-response curve, with maximal cAMP levels attained with $10^{-5}$ to $10^{-4} \mathrm{M}$ DA and an $\mathrm{EC}_{50}$ of $1.30 \mu \mathrm{M}$.

Both receptors had similar agonist profiles, and the typical vertebrate D1-like receptor agonist SKF-38393 was ineffective. Experiments with antagonists revealed that BmDopR1 has D1-like features. However, the pharmacology of BmDopR2 was distinct from that of D1-like receptors; the typical vertebrate D1-like receptor antagonist SCH-23390 was less potent than the nonselective antagonist flupenthixol and the D2-like receptor antagonist chlorpromazine. The rank order of the activities of several antagonists for BmDopR1 and BmDopR2 was more similar to that of Drosophila melanogaster DA receptors than Apis mellifera DA receptors. These data suggest that DA receptors are potential targets for specific insecticides or insectistatics.

\section{Concluding remarks}

In this study, we constructed the cell systems stably expressing TA, OA, and DA receptors cloned from B. mori and characterized functions, ligand-bindig sites, and pharmacological properties of the receptors. The use of the stable expression systems would facilitate the study of structure-activity relationships of compounds and the discovery of specific receptor ligands. Such study will aid in the development of novel agrochemicals that interact with biogenic amine receptors specifically. 